\title{
SWSDM: SOCIAL WEB SERVICE DISCOVERY MODEL
}

\author{
Amal Hafsi, Youssef Gamha and Lotfi Ben Romdhane \\ MARS Research Laboratory LR17ESO5, Higher Institute of Computer Science and Telecom (ISITCom), \\ University of Sousse, Tunisia
}

\begin{abstract}
The number of services is growing at an explosive rate, creating great challenges in efficiently extracting the target services for users. Moreover, during recent years, social networks have emerged with the progress of the Web 2.0 and the popularity of social networks websites like Twitter and Facebook has increased rapidly. Adding social dimension in the Service-Oriented Architecture have facilitated web services discovery. Thus, web services can be treated as an active entities by being part of social networks. This paper proposes a social web services discovery model relying on the integration of social web services, and more social users in the discovery process. We aim to involve both users and web services in the discovery process, instead of focusing only on the web services. The results of using our framework show that the discovery process becomes easier if we take advantage of the relations among web services in their own social networks and linking both services and users in the same network.
\end{abstract}

\section{KEYWORDS}

Social Networks, Social Web Services, Social Web Services Discovery

\section{INTRODUCTION}

The Service-Oriented Computing (SOC) paradigm promotes developing new applications and solutions by using services as fundamental elements. A few years ago, SOC has come into view as a new paradigm for assembling complex Web applications Papazoglou (2003). To build the service model, SOC extends a service oriented architecture (SOA), which is a logical way of reorganizing software applications and infrastructure into a set of interacting services. As one of the most popular model for implementing a SOA system, web services are active software programs that are mainly designed to support machine to machine interactions through the web. The growing use of web services changes the World from the Internet of web pages to the Internet of services (IoS) Pistore et al. (2009). Thus, IoS will offer services for all areas of life and business, such as online banking, travel tickets, and so on. SOA, Web2.0 and Web3.0 are the Building blocks for the IOS. The web service discovery is one of the most challenging problems in service oriented computing. The growing number of web services makes the task of searching for relevant services by a service requester harder. The providers publish their services using the Universal Description Discovery and Integration registry (UDDI). However, UDDI and search engines (like seekda ${ }^{1}$ return low precision results mainly, i.e., they return a large number of services to the users Rajmohan et al. (2011), Maamar et al. (2011), and the lack of dynamics, i.e., the user must query the registry regularly to look for the appropriate services Dustdar \& Treiber (2005). Moreover, in a registry, the web service is treated as an isolated element Maamar et al. (2011). In fact, available registries do not capture how each web service was interacting with peers. Recently, with the advent of social Web, many web service discovery model, which is based on web services social networks, have been proposed Maamar et al. (2011); Fallatah et al. (2014). Integrating social concepts in service computing to build social web services (SWSs) has enhanced the web service discovery. An SWS is a service that lives in an interlinked network of services, where services can (i) collaborate to satisfy complex users needs, (ii) recommend the services to replace it, and (iii) compete with each other to be selected. A community exists that mixes social networks of web services and social networks of users, exploiting the potential of social networks to include users to be interactive entities, opens the door to new discovery schemes Fallatah et al. (2014). In recent years, some approaches Fallatah et al. (2014); Kalaï et al. (2018), which consider the users social relationships and

\footnotetext{
${ }^{1}$ https://seekda.com
} 
profile, have been proposed. Thus, the integration of users and services into a common social network, like SOAF (Service- Of- A- Friend) Network Treiber et al. (2009), has been a new line of research. In some works like Fallatah et al. (2014), the system explores all the social links of the service requester in order to verify if any of his/her relations had invoked a service that satisfy his/her needs. However, the majority of service requester would to be suggested by a web service used by a friend who shares the same interests with him, more than another friend in his/her social network. So, it is necessary to take into account the interest similarity between friends. Moreover, a friend, who used services in the same domain of the users query, can suggest a service similar to the needs of service requester. Thus, it is necessary to consider this information in order to provide a better suggestion quality. In others studies Kalaï et al. (2015), the approach does not consider all the social relations but it selects only the similar ones using the advantage of Similarity Degree measure. This measure is calculated based on the number of common friends between service requester and his/her friends. This measure seems non reliable and doesn't reflect the real similarity between friends. However, all of them have neglected the impact of time in service discovery. In fact, the service, which is used by a user two years ago for example, can be unavailable.

In our research work, we propose a new discovery process for SWSs using web services social networks, the links that can exist among users in their own social networks and the links between both users and web services. For instance, assume a user wants to spend the holidays in another city. He must travel by plane and book a taxi to the hotel. We launched the query which is a composite service: Buy a plane ticket, book a taxi and a hotel room. Our System should identify the most relevant services in response to the requester's query. This paper is organized as follows, section 2 discusses related work, section 3 highlights our model, and the experimental evaluations will be presented in section 4 . The conclusion is given in section 5 .

\section{RELATED WORK}

Several approaches have been introduced in the literature. Maamar et al. (2009) present a novel model that captures relationships between web services as they interact with each other using social networks. They used recommender systems for web services discovery based on social networks by proposing two scenarios: the recommendation based associations to enrich the process of composition with additional web services, and the recommendation-based associations, with focus on the robustness that allows services to select replacement peers if it is unavailable. In Maamar et al. (2011), the authors presented LinkedWS: A service discovery model based on the interaction between web services in the context of social networks. They defined two social networks types: a) a collaboration network if web service collaborates with other peers in order to answer complex users needs and b) recommendation network is divided into partnership and robustness networks. In the robustness networks, web services can designate other services to substitute them if they cannot satisfy the user's request. In the partnership networks, web services are able to recommend other services to participate in composition. The authors combine two paradigms: social computing and SOC in order to give birth to SWSs.

In Maamar et al. (2012), the authors present a four-step method to engineer SWSs. The questions that the method addresses are: what relations between web services exist, what social networks correspond to these relations, how to build social networks of SWSs, and what social behaviors SWSs can exhibit. Over the past few years, several works Maamar et al. (2007, 2013), Metrouh \& Mokhati (2013) have been introduced to propose a social dimension based representation for web services. In Metrouh \& Mokhati (2013), the authors introduced an approach for the SWSs discovery process. In fact, they defined two types of associations between web services in the social networks. The first associations is collaboration, it introduces the concept of service community. Recommendation-based associations, the second type, allow web service to propose other services to participate in a composition. Maamar et al. (2011) proposed to incorporate users and services in a same social network to compose, execute, and monitor web services. More recently, Fallatah et al. (2014) have proposed a new social network-based framework for analyzing the role and influence of users and web services in the discovery process. They proposed a new discovery process for SWSs using the social structure of those services and also benefiting from the links that can exist among users in their own social networks. The authors used a three-step engineering method to construct users and web services social networks: identifying social networks components, establishing relationships between users and web services and describing service and user aspects. Kalaï et al. (2015) presented a web service decentralized discovery approach based on user query and his social ego-centric network. The discovery process extracts the invoked satisfactory web services of his 
best friends to generate results. They integrated the users and their invoked satisfactory services into the same structure of their social networks. In Corbellini et al. (2017), the authors propose an approach to discover SWSs that operates on graphs with user-service relationships and clusters socially similar services which are determined by exploiting explicit relationships and mining implicit relationships in the graph. Also, Duan \& Tian (2017) present a collaborative service discovery that uses social link to identify the relationships between users and services. The social link is decomposed into two factors: clustering link and preference link. A user's Preference Link is defined as the set of services invoked by him/her. Clustering Link (CL) contains two subclasses: Service Clustering Link (SCL) and User Clustering Link (UCL). SCL is the set of services and their similarities, which is based on similarity functions and similarity names. UCL is the set of users and their similarities: similarity between two users is determined by comparing their models.

In our work, we propose a SWSs model SWSDM which is based on web services social network and user's profile and takes into account web service invocation date.

\section{BIPARTITE-BASED DISCOVERY APPROACH}

\subsection{Architecture Overview}

Our approach for the SWSs discovery process based on a bipartite graph, as illustrated in Figure 1, has an input (i) the user's query which can be an atomic service or a composite service, and (ii) the social network of users, and (iii) the web service repository.

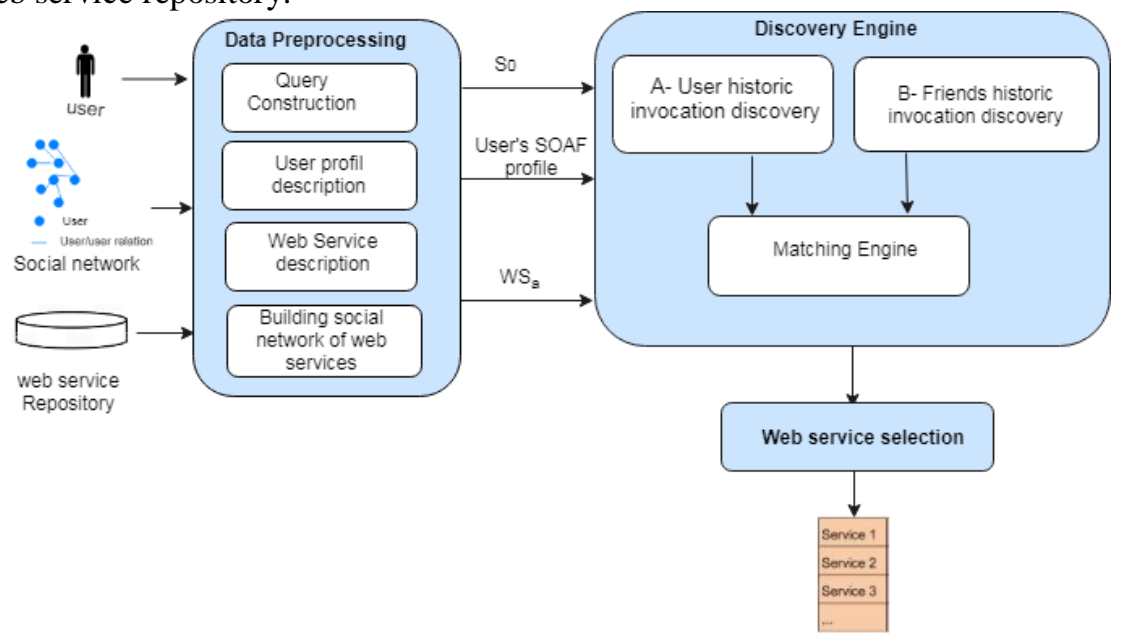

Figure 1. Architecture of our SWSDM

A social network of users is viewed as a graph $\mathrm{SN}_{\mathrm{u}}\left(\mathrm{U}, \mathrm{E}_{\mathrm{u}}\right)$ where:

- $\quad \mathrm{U}$ is the set of nodes which represents the users. Each user's node is described by a profile.

- $\mathrm{E}_{\mathrm{u}}$ is the set of edges which represent the social relationships between those nodes.

Our discovery process performs two components: data preprocessing and discovery engine. The first component includes four steps: query construction, user profile description, web service description and building social network of web services. These data are introduced to the module of discovery engine which includes two steps: (a) User historic invocation discovery process based on the social profile and the egocentric collaboration social networks of services and (b) friends historic invocation discovery process based on social relations between users. The set of web services discovered is sent to the matching engine to evaluate the similarity with user's query in term of functionality.

\subsubsection{Data Preprocessing}

This section introduces the data processing module for a given data sets, which includes four main phases, namely query construction, user profile description using the Service of a Friend ontology SOAF Treiber et al. (2009), web service description, and building social network of web services. 
- Query construction: The user query are described with WSDL-S document Akkiraju (2005). A WSDL-S Web Service Description is a WSDL description enriched with semantic annotation which can be references to concepts defined in external ontologies Akkiraju (2005). In this step, we preprocess the content of each service in the service repository of the test collection to build a WSDL-S description. User query is modeled as following:

Definition 1. User Query (S0) is described by: $\mathrm{S} 0=\left(\left\{\mathrm{WS}_{\mathrm{a}}\right\}, \sigma, \mathrm{D}\right):\left\{\mathrm{WS}_{\mathrm{a}}\right\}$ is the set of atomic services, $\sigma$ is a control operator that define the composition, $\mathrm{D}$ is the set of domain for atomic services.

- User profile description: In this step, we generate a profile for each user in the social networks; it contains information about the user's characteristics: the interests of items (e.g. music, football, etc.), the links that define the social relationships between users, and the past interactions between the user and his web services which were used previously. User's profile is based on the SOAF model. User profile is modeled as following:

Definition 2. User profile ( $\subset \subset \mathrm{U}$ ) is modeled by a triplet $\mathrm{u}=(\mathrm{I}, \mathrm{S}, \mathrm{F})$ : $\mathrm{I}$ is a user interests set, $\mathrm{S}$ is the set of previously used web services and $\mathrm{F}$ is the user social relationships sets.

- Web service description: it corresponds to the semantic description of web services, by detailing these functional properties. Relevant services and user query are semantically described with WSDL-S. Due to lack of such data sets, we create a WSDL-S file for each web service in the repository. A web service in our model is defined respectively by the following tuple:

Definition 3. An atomic web service is described by: $\mathrm{WS}_{\mathrm{a}}=(\mathrm{I}, \mathrm{O}, \mathrm{E}, \mathrm{P}, \mathrm{d})$ : $\mathrm{I}$ is the service input parameters, $\mathrm{O}$ is the service output parameters, $\mathrm{E}$ is the finite set of effects, $\mathrm{P}$ is the finite set of Preconditions and $\mathrm{d}$ is the service domain.

- Building web services social network: Establishing social networks of SWSs consist of six steps Maamar et al. (2011). The aim of developing SWSs is to discover all peers' service that it likes to work with when building compositions, and collaboration and substitution without using screening registries. In this paper, we focus on the collaboration social network.

Step 1 - Determining social network components

Web services social network have two components: the nodes represent web services, and the edges represent the links between those nodes. A collaboration edge means that web services have the capacity to work together on complex user requests. Formally, a Social Network $\mathrm{SN}_{\mathrm{Sws}}$ of a web Service is a couple: $\mathrm{SN}_{\mathrm{Sws}}=(\mathrm{N}, \mathrm{E})$ where $\mathrm{N}$ and $\mathrm{E}$ are respectively, the set of Nodes and Edges.

Step 2 - Matching analysis of web services

To create a collaboration relationship between web services, we match their respective functional properties. We use an approach that evaluates the Complementary degree (DC) between two web services Liu et al. (2009).

Step 3 - Social Network Management

First of all, we have to select a web service root, the selection is random since any web service that will join the future social network can be treated as a root. The life-cycle of a social network is as following:

- $\quad$ Building stage: we choose randomly a root.

- Exploitation stage: The aim of this stage is looking for potential collaborators to the root.

- $\quad$ Expansion state: Each service that will be added to the social network is treated as a root.

Step 4 - Initial evaluation of weights

The complementary degree between two SWSs is considered as the first value of an edge weight between them. This value (1) will update as soon as new SWSs are discovered.

$$
\begin{aligned}
& \text { WEt0(SWSi, SWSj) = DC(SWSi, SWSj) } \\
& \text { Step } 5 \text { - Navigation through the social network }
\end{aligned}
$$

Social network collaboration allows users to identify a SWS as an entry point in the network and then navigate along social links to look for collaborators deemed necessary for composition.

Step 6 - Ongoing edge weight evaluation

The ongoing evaluation happens by updating the edge weights each time a collaborative peer is discovered. This equation evaluate the weights in a collaboration social network using reward-based prices Yu et al. (2004): 
$\mathrm{WEt}+\delta \mathrm{t}(\mathrm{SWSi}, \mathrm{SWSj})=W E t(S W S i, S W S j)+\alpha \times\left(\frac{\mid \mathrm{SWSj} \text { selection } \mathrm{t}+\delta \mathrm{t} \mid}{\mid \mathrm{SWSi} \text { collaboration } \mathrm{t}+\delta \mathrm{t} \mid}-\mathrm{WEt}(\mathrm{SWSi}, \mathrm{SWSj})\right)$

where $\alpha €[0,1], \delta t$ represents the update period, $\mid S W S_{j}$ selectiont ${ }_{j} \mid$ determine how often $\mathrm{SWS}_{\mathrm{j}}$ and $\mathrm{SWS}_{\mathrm{i}} \mathrm{Were}$ engaged in collaboration following the use of SWSi's collaboration social network at time $t$, and $\mid \mathrm{SWS}_{\mathrm{i}}$ collaboration $_{\mathrm{t}} \mid$ is the number of times that $\mathrm{SWS}_{\mathrm{i}}$ was engaged in collaborations at time $\mathrm{t}$.

\subsubsection{Discovery Engine: User Historic Invocation Discovery}

Our discovery engine performs a (a) User historic invocation discovery process, which is the first component that returns to the current user web service(s) based on his previous experience and the egocentric collaboration social networks, and (b) Friends historic invocation discovery process, the second component that offers to the requester web service(s) according to his friendship relations. The objective of discovery engine is to evaluate a Degree of Similarity (DS, Eq. (4)) between two services ws $\mathrm{s}_{\mathrm{i}}$ and $\mathrm{ws}_{\mathrm{j}}$ using a Matching Score (MS, Eq. (3)). Two web services $w_{s_{i}}$ and $w_{s}$ are similar when $w s_{i}$ 's inputs, wsi's outputs, $\mathrm{ws}_{\mathrm{i}}$ 's preconditions, and $\mathrm{ws}_{\mathrm{i}}$ 's effects match respectively $w_{s_{j}}$ 's inputs, $w s_{j}$ 's outputs, $w s_{j}$ 's preconditions, and $w s_{j}$ 's effects. The matching score (MS) between the two concepts $\mathrm{c}_{\mathrm{wsi}}$ and $\mathrm{c}_{\mathrm{wsj}}$ in the same profile category is a function of (i) $l$ is the number of edges from $c_{w s i}$ to $c_{w s j}$ in the ontology, (ii) $h$ is the depth of $c_{w s i}$ in the ontology, and (iii) $d$ is the local semantic density of $\mathrm{c}_{\mathrm{wsi}}$ and $\mathrm{c}_{\mathrm{wsj}}$ Liu et al. (2009). We compute the matching score as follows:

$$
M S(c w s i ; c w s j)=f 1 \times f 2 \times f 3
$$

where $f 1=e \alpha l$ with $\alpha$ as a constant, $f 2=\frac{\mathrm{e} \beta \mathrm{h}-\mathrm{e}-\beta \mathrm{h}}{\mathrm{e} \beta \mathrm{h}+\mathrm{e}-\beta \mathrm{h}}$ with $\beta$ as a smoothing factor, and $\mathrm{f} 3=\frac{\mathrm{e} \lambda \mathrm{l}-\mathrm{e}-\lambda \mathrm{l}}{\mathrm{e} \lambda \mathrm{l}+\mathrm{e}-\lambda \mathrm{l}}$ with $\lambda$ as another smoothing factor. We calculate the degree of similarity $([0,1])$ between two services ws $\mathrm{s}_{\mathrm{i}}$ and $\mathrm{ws}_{\mathrm{j}}$ using the weighted average of the matching scores between each concept pair in the profile categories:

$$
D S(w s i, w s j)=\frac{\sum_{k} W k \times \mathrm{MS}(\mathrm{c}(\mathrm{wsi}) \mathrm{k}, \mathrm{c}(\mathrm{wsj}) \mathrm{k})}{\sum_{k} w k}
$$

where $\mathrm{k}$ is the total number of similar concepts and $\mathrm{W}_{\mathrm{k}}$ is the weight associated with the matching score between a pair of concepts. In this paper, we will focus on the first step of our model, user historic discovery process. This section is composed of two parts: a part to describe the user historic invocation discovery process, and a second part to present the proposed algorithm.

\section{Description}

Formally, we define our bipartite graph $\mathrm{G}$ as a triplet $(\mathrm{U}, \mathrm{N}, \mathrm{R})$, where $\mathrm{U}$ is the nodes set of the users social network, $\mathrm{N}$ is the nodes set of the web services social network and $\mathrm{R}$ represent the links between those nodes which show that a user has previously invoked a web service.

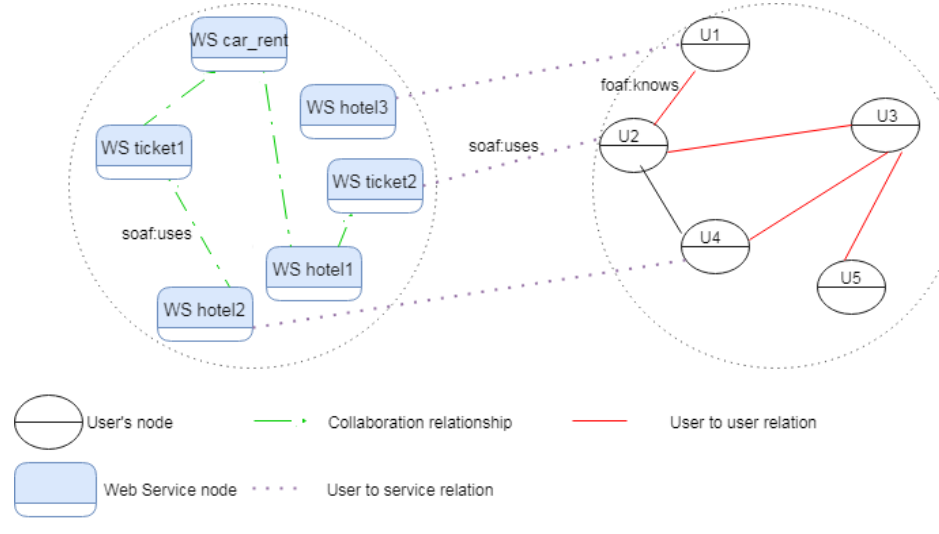

Figure 2. Instance of our bipartite graph

Figure 2 presents an instance of our bipartite graph. In this instance, there are six web services and five users.

This first component uses the social profile of the current user in order to extract all the web services previously invoked in a definite time interval and evaluated as "very satisfied". In fact, we assume that the user 
evaluates the service after using according to four modalities: very satisfied / not very satisfied / not satisfied. In our approach, we take into account the concept of time to extract only the web services recently invoked, for example a web service which invoked two years ago can be not yet functional. Next, the discovered web services are sent to the matching engine to verify the satisfaction of the query. If the request is not satisfied, we extract all the SWs which belong to the egocentric collaboration network of each discovered web service. An egocentric collaboration network can be defined as a sub-network consisting solely of a web service and all of its direct links in the collaborative social network. Then, a matching between the query and each web service discovered is done.

Algorithm of SWSs discovery

In this section, we present two algorithms named User historic Discover and Extract Invoked SWS which implemented the first step of our model. The details of the algorithm Extract Invoked SWS are as following . The purpose of this algorithm is extracting from the profile of the current user all the satisfied web services which are invoked previously by him in a period of time $\Delta \mathrm{t}$. This algorithm allows for the following tasks in order: (i) we extracted the list of invoked web services by applying the ExtractSWS function (line 1), (ii) we loop over web services set, if the service is recently invoked, we add it to the list (line 2-6), (iii) we return the list of invoked services.

Algorithm 1:Extract Invoked SWS

Input: $\mathrm{u}$ : SOAF document, $\Delta \mathrm{t}$ : period of time;

Output: SWS P;

Begin

1: List SWSI = ExtractSWS (u);

2: for each SWSi in List SWSI do

3: if (Connection date $(\mathrm{SWSi}) \subset \Delta \mathrm{t})$ then

4: SWS P = SWSi;

5: end if, end for

6: return SWS P;

7: End

In the following algorithm (See Algorithm 2), we firstly apply the Extract Invoked SWS function (lines 1). Then, we match the user's query S0 with each extracted web service in SWS list (lines 2 - 6). If the query is not satisfied (line 7), for each discovered web service $\mathrm{SWS}_{\mathrm{P}}$, we extract all the web services which belong to his egocentric collaboration network (line 8 - 10), and we match them with the query. The set of discovered web services and a Boolean variable to indicate the satisfaction of the query are returned.

Algorithm 2: User historic Discover

Input: u: SOAF document, S0: Request, $\Delta \mathrm{t}$ : period of time;

Output: $\mathrm{SWS}_{\mathrm{disc}}$, Sat;

Begin

1: SWS_P $\leftarrow$ Extract Invoked SWS $(\Delta \mathrm{t}, \mathrm{u})$;

2: for each SWS $_{P}$ in SWS_P do

3: for $\mathrm{i}=1$ to $\mathrm{k}$ do

4: $\mathrm{SWS}_{\mathrm{disc}}$, Sat $\leftarrow$ matching $\left(\mathrm{SWS}_{\mathrm{P}}, \mathrm{S}_{\mathrm{a}}[\mathrm{i}]\right)$;

5: end for, end for

6: if Sat $=$ False then

7: for each SWSP in SWS P do

8: SWS R $\leftarrow$ Extract Neighbors (SWSP);

9: end for

10: for each $S W S_{R}$ in SWS_R do

11: for $\mathrm{i}=1$ to $\mathrm{k}$ do

12: $\mathrm{SWS}_{\mathrm{disc}}$, Sat $\leftarrow$ matching $\left(\mathrm{SWS}_{\mathrm{R}}, \mathrm{S}_{\mathrm{a}}[\mathrm{i}]\right)$;

13: end for, end for, end if

14: return $\mathrm{SWS}_{\text {disc, }} \mathrm{S}$;

15: End 


\section{IMPLEMENTATION AND EXPERIMENTS}

SWSDM is written in Java v10.0.1 and run under the operating system Windows 7. Through this evaluation step, we try to show the importance of using the social network of web services to answer complex user query. Due to lack of data sets that integrates the users and the web services, we used three independent data sets. The first is the set of users who are linked to a social network. We used the SNAP STANFORD ${ }^{2}$ real data sets "Epinions social network" which contains 75879 users and 508837 links. SWSD uses as second data set the service repository of test collection SemWebCentral ${ }^{3}$. There are 1083 semantic web services and 42 requests advertised with OWL-S 1.1 and SAWSDL 1.1. There are seven service domains: Education, Medical, Food, Travel, Communication, Economy, and Weapon. We generate the WSDL-S file for each service. We used a third artificial dataset that contains for each users, invoked services, evaluation and date of invocation. Experiments were run for 20 query and 3 users (we have chosen users who have 5 to 15 services in the profile) in order to evaluate the query satisfaction. Table 1 presents a comparison between two approaches: social profile based approach which uses only the profile of the user, and egocentric collaboration network based approach (the first process of SWSDM) which uses not only the social profile of the user but also the egocentric collaboration social network of each web service discovered. The preliminary results show that, when the social network of web services is used, the percentage of getting a satisfied query is higher than in the case of using only the user's social profile.

Table 1. Comparison between two approaches

\begin{tabular}{lccc}
\hline & \#SW in user profile & \#satisfied query & Query satisfaction percentage \\
\hline \multirow{2}{*}{ Social profile based } & 5 & 4 & $20 \%$ \\
& 10 & 5 & $25 \%$ \\
& 15 & 7 & $35 \%$ \\
Egocentric collaboration & 5 & & \\
network based & 10 & 13 & $65 \%$ \\
& 15 & 15 & $75 \%$ \\
& & 15 & $75 \%$ \\
\hline
\end{tabular}

\section{CONCLUSION}

This paper presents an approach to discover web services that takes advantage of using the metaphor of social networks. The blend of social computing with service-oriented computing gives birth to SWSs. Thanks to this blend, web services are not handled as isolated elements but they know the peers with whom they worked in the past and with whom they would like to work in the future. Different types of networks that connect web services together exist. These networks include collaboration, substitution, and competition. In our approach, we focused only on the collaboration social network. We detailed our discovery process which is based on two components: data preprocessing and discovery engine. The first refers to the transformations applied to our data sets before feeding it to the second component. Discovery engine performs two processes. In this paper, we presented the first process. Our method takes advantage of using web services social networks to enhance the discovery process. The experimentation indicates that the number of satisfied queries returned by our egocentric collaboration network based approach is more than the number returned by the social profile based approach. In order to satisfy more the complex user's queries, in our future works, we will present the friend's historic invocation discovery process which pushes the idea of using both the users and web services social network.

\footnotetext{
${ }^{2}$ SNAP STANFORD, www.snap.stanford.edu

${ }^{3} \mathrm{http}: / /$ semwebcentral:org/frs
} 


\section{REFERENCES}

Akkiraju, R. a. F. J. a. M. J. A. a. N. M. a. S. A. P. a. V. K., 2005. Web service semantics-wsdl-s.

Corbellini, A. et al., 2017. Mining Social Web Service repositories for social relationships to aid service discovery. s.l., s.n., pp. 75-79.

Duan, L. \& Tian, H., 2017. Collaborative Web Service Discovery and Recommendation Based on Social Link. Future Internet, Volume 9, p. 63.

Dustdar, S. \& Treiber, M., 2005. A view based analysis on web service registries. Distributed and Parallel Databases, Volume 18, pp. 147-171.

Fallatah, H., Bentahar, J. \& Asl, E. K., 2014. Social network-based framework for web services discovery. s.l., s.n., pp. 159-166.

Kalaï, A., Zayani, C. A. \& Amous, I., 2015. User's Social Profile--Based Web Services Discovery. s.1., s.n., pp. 2-9.

Kalaï, A. et al., 2018. Social collaborative service recommendation approach based on user's trust and domain-specific expertise. Future Generation Computer Systems, Volume 80, pp. 355-367.

Liu, M., Shen, W., Hao, Q. \& Yan, J., 2009. An weighted ontology-based semantic similarity algorithm for web service. Expert Systems with Applications, Volume 36, pp. 12480-12490.

Maamar, Z. et al., 2013. From Social Web Services to Social Communities of Web Services. Advances in Information Sciences (AIS), Volume 1, pp. 22-37.

Maamar, Z., Faci, N., Sheng, Q. Z. \& Yao, L., 2012. Towards a user-centric social approach to web services composition, execution, and monitoring. s.l., s.n., pp. 72-86.

Maamar, Z. et al., 2011. Using social networks for web services discovery. IEEE internet computing, Volume 15, pp. 48-54.

Maamar, Z. et al., 2011. Towards a method for engineering social web services. In: Engineering Methods in the Service-Oriented Context. s.1.:Springer, pp. 153-167.

Maamar, Z., Hacid, H. \& Huhns, M. N., 2011. Why web services need social networks. IEEE Internet Computing, Volume 15, pp. 90-94.

Maamar, Z. et al., 2007. Web Services Communities-Concepts \& Operations.. s.1., s.n., pp. 323-327.

Maamar, Z., Wives, L. K., Badr, Y. \& Elnaffar, S., 2009. Even web services can socialize: A new service-oriented social networking model. s.l., s.n., pp. 24-30.

Maamar, Z. et al., 2011. LinkedWS: A novel Web services discovery model based on the Metaphor of "social networks". Simulation Modelling Practice and Theory, Volume 19, pp. 121-132.

Metrouh, A. \& Mokhati, F., 2013. Social web services discovery: A community-based approach. s.l., s.n., p. 275.

Papazoglou, M. P., 2003. Service-oriented computing: Concepts, characteristics and directions. s.1., s.n., pp. 3-12.

Pistore, M., Traverso, P., Paolucci, M. \& Wagner, M., 2009. From Software Services to a Future Internet of Services.. s.1., s.n., pp. 183-192.

Rajmohan, R., Padmapriya, N. \& Jayakumar, S. K. V., 2011. A survey on problems in distributed UDDI. International Journal of Computer Applications, Volume 36, pp. 975-8887.

Treiber, M., Truong, H.-L. \& Dustdar, S., 2009. Soaf--design and implementation of a service-enriched social network. s.l., s.n., pp. 379-393.

Treiber, M., Truong, H.-L. \& Dustdar, S., 2009. Soaf--design and implementation of a service-enriched social network. s.l., s.n., pp. 379-393.

Yu, B., Li, C., Singh, M. P. \& Sycara, K., 2004. A dynamic pricing mechanisms for $22 p$ referral systems. s.l., s.n., pp. 1426-1427. 